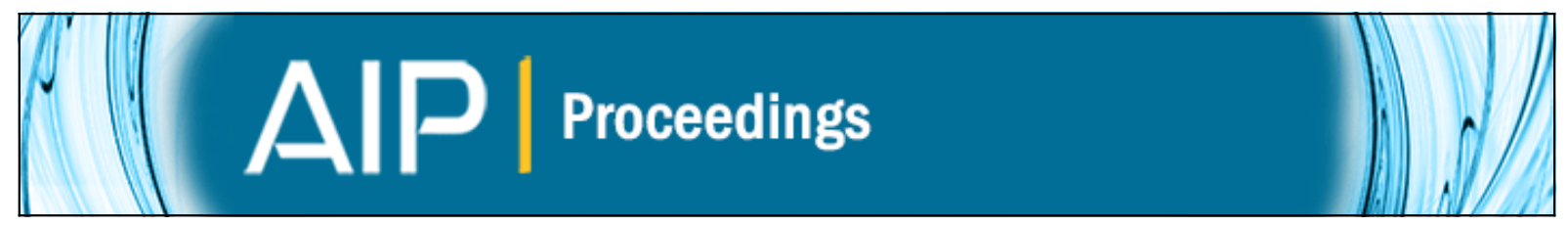

\title{
Nanotechnology and textiles engineered by carbon nanotubes for the realization of advanced personal protective equipments
}

Antonio Andretta, Maria Letizia Terranova, Teresa Lavecchia, Stefano Gay, Alfredo Picano, Alessandro Mascioletti, Daniele Stirpe, Cristian Cucchiella, Eddy Pascucci, Giovanni Dugnani, Davide Gatti, Giuseppe Laria, Barbara Codenotti, Giorgio Maldini, Siegmar Roth, Daniele Passeri, Marco Rossi, and Emanuela Tamburri

Citation: AIP Conference Proceedings 1603, 71 (2014); doi: 10.1063/1.4883044

View online: http://dx.doi.org/10.1063/1.4883044

View Table of Contents: http://scitation.aip.org/content/aip/proceeding/aipcp/1603?ver=pdfcov

Published by the AIP Publishing

\section{Articles you may be interested in}

The effect of firefighter personal protective equipment on auditory thresholds

J. Acoust. Soc. Am. 134, 4228 (2013); 10.1121/1.4831532

A carbon nanotube based ammonia sensor on cotton textile

Appl. Phys. Lett. 102, 193104 (2013); 10.1063/1.4805025

Computational Nanotechnology with Carbon Nanotubes and Fullerenes

Comput. Sci. Eng. 3, 42 (2001); 10.1109/5992.931903

Nanotechnology of nanotubes and nanowires: From aligned carbon nanotubes to silicon oxide nanowires AIP Conf. Proc. 442, 29 (1998); 10.1063/1.56529

Personal Protection

Noise Control 2, 37 (1956); 10.1121/1.2369176 


\title{
Nanotechnology and Textiles Engineered by Carbon Nanotubes for the Realization of Advanced Personal Protective Equipments
}

\author{
Antonio Andretta ${ }^{1, \mathrm{a})}$, Maria Letizia Terranova ${ }^{2}$, \\ Teresa Lavecchia ${ }^{2}$, Stefano $\mathrm{Gay}^{2}, \mathrm{Alfredo} \mathrm{Picano}^{3}$, Alessandro Mascioletti ${ }^{3}$, \\ Daniele Stirpe ${ }^{3}$, Cristian Cucchiella ${ }^{4}$, Eddy Pascucci ${ }^{4}$, Giovanni Dugnani ${ }^{5}$, \\ Davide Gatti ${ }^{5}$, Giuseppe Laria ${ }^{6}$, Barbara Codenotti ${ }^{7}$, Giorgio Maldini ${ }^{8}$, Siegmar \\ Roth $^{9}$, Daniele Passeri ${ }^{10}$, Marco Rossi ${ }^{10}$ and Emanuela Tamburri ${ }^{2}$ \\ ${ }^{1}$ Klopman International S.R.L., Via Mola dei Frati, 03100 Frosinone, Italy \\ ${ }^{2}$ Dipartimento di Scienze e Tecnologie Chimiche, Minima lab, Università di Roma 'Tor Vergata', Via della Ricerca \\ Scientifica, 00133 Roma, Italy \\ ${ }^{3}$ Labor - Industrial Research Lab, Via Giacomo Peroni, 386 C/O Tecnopolo Tiburtino, 00131 Roma, Italy \\ ${ }^{4}$ InfoSolution S.p.A, Via Zoe Fontana 10, 00131 Roma, Via Luigi Cadorna 67, 20090 Vimodrone (MI), Italy \\ ${ }^{5}$ Tpa Spa - Tecnologie e Prodotti per l'Automazione, Via Carducci 221, 20099 Sesto San Giovanni (MI), Italy \\ ${ }^{6}$ Centre of Research for Pure and Applied Mathematics, via Giovanni Paolo II 132, 84084 Fisciano (SA), Italy \\ ${ }^{7}$ Lavanderie dell'Alto Adige S.p.A., via Nazionale 55, 39040 Ora (BZ), Italy \\ ${ }^{8}$ Meta System S.p.A., Via Galimberti 8, 42124 Reggio Emilia, Italy \\ ${ }^{9}$ SINEUROP-Nanotech GmbH, TBC Kernerstrasse 34, 70182 Stuttgart, Germany \\ ${ }^{10}$ Dipartimento di Scienze di Base ed Applicate per l'Ingegneria and CNIS, Sapienza Università di Roma, Via \\ Scarpa 16, 00161 Roma, Italy

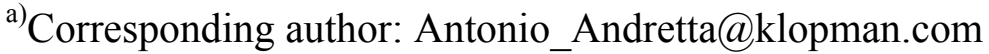

\begin{abstract}
Carbon nanotubes (CNT) and CNT-based active materials have been used to assemble the gas sensing unit of innovative platforms able to detect toxic atmospheres developing in confined workplaces. The main goal of the project was to realize a full-featured, operator-friendly safety detection and monitoring system based on multifunctional textiles nanotechnologies. The fabricated sensing platform consists of a multiple gas detector coupled with a specifically designed telecommunication infrastructure. The portable device, totally integrated in the workwear, offers several advantages over the conventional safety tools employed in industrial work activities.
\end{abstract}

Keywords: gas sensor, multifunctional textiles, carbon nanotubes, safety, workwear PACS: 07.07.Df, 89.60.Ec, 61.48.De

\section{INTRODUCTION}

Successful protection of health and safety of workers on the workplace requires adequate infrastructure and equipment, based on effective and reliable technologies. Several types of industrial activities, such as working in a confined space, welding, and many other routine procedures for plant maintenance procedures, are highly associated with potential dangers due to the development of dangerous atmospheric conditions. Among the possible scenarios, there are oxygen deficiencies, poisoning by toxic gas exposure and/or explosions. An accurate and rapid detection of dangerous atmospheric conditions is required to protect workers from health and safety risks arising from activities in hazardous environments. However, the gas monitoring devices set out by present regulations are rather bulky and expensive. Further disadvantages are moreover associated with such instruments, such as the lack of accuracy, the 
tendency to saturate, the energy high consumption, the incidence of false alarms. There is therefore a pressing need of technological improvements for portable detectors with fast response for the identification of dangerous gaseous.

In this context it was felt worthwhile to develop an innovative sensing platform, based on new technical and multifunctional textiles, for the protection of workers and emergency services exposed to safety issues [1]. Six enterprises with the support of two Universities decided to embark on a challenging research encompassing the fields of material science and nanotechnology. The project, launched with the name "SENSATIONAL" and funded by the Minister for Economic Development (Italy), was aimed at the design and fabrication of a platform made up by 2 main components, namely a technical textile with multi-gas sensing features, and a telecommunication support. Figure 1 illustrates the scheme of the SENSATIONAL platform. In the following the main characteristics of the various components are briefly illustrated.

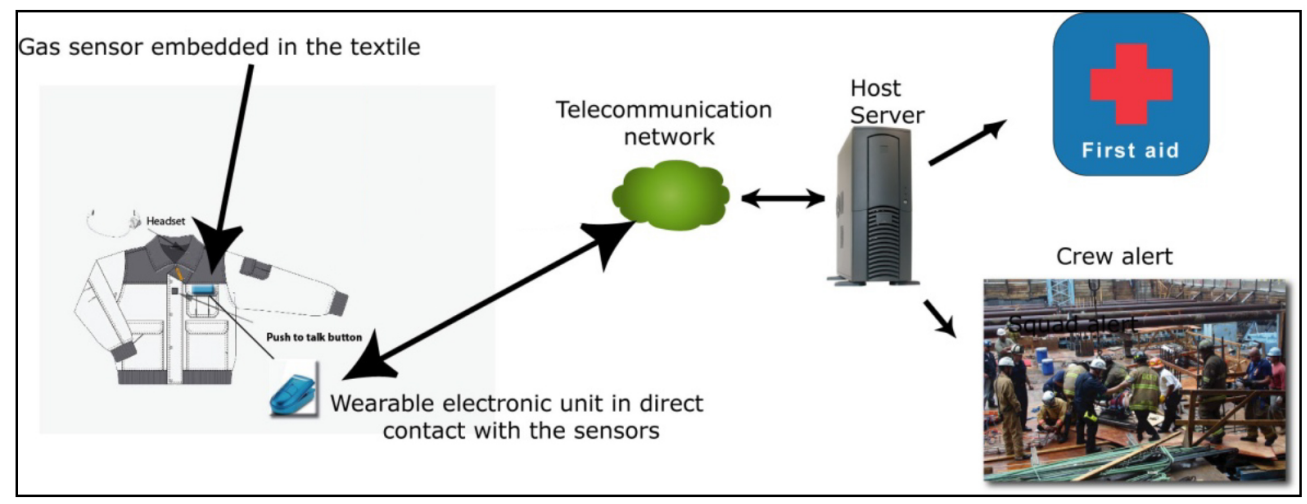

FIGURE 1. Scheme of the SENSATIONAL platform.

\section{THE SENSOR}

The sensor units are fabricated exploiting the amazing properties of carbon nanotubes as active sensing material. These nanomaterials, characterized by excellent chemical-physical, mechanical and electronic properties, show large effective surface area $\left(-1600 \mathrm{~m}^{2} / \mathrm{g}\right)$, which increases the probability of gas-nanotube interactions, and are thus ideal candidates for gas sensing application. In the present application the property of the nanotubes to change dramatically their conductance upon exposure to gaseous species was exploited. Chemicals induced perturbations of the nanotube conductance gives direct information, easy to read-out and to interface with conventional electronic architectures. Compared with conventional solid-state sensors, that typically operate at temperatures over $400^{\circ} \mathrm{C}$, and conducting polymer-based sensors, that provide only limited sensitivity, sensing devices assembled with singlewall nanotubes can exhibit high sensitivity and fast response time at room temperature. Moreover the outstanding mechanical properties of CNTs, combining a high Young's modulus and intrinsic flexibility due to the high aspect ratio, make them the ideal candidate for the integration in textiles.

Project's activities have been carried out following a research line that had already shown the ability of the nanotube based sensors to detect at room temperature ppm or sub-ppm levels of gas molecules $\left(\mathrm{NO}_{\mathrm{x}}, \mathrm{NH}_{3}, \mathrm{CO}, \mathrm{H}_{2} \mathrm{~S}\right)$ in some hundreds of microseconds [2-4].

The nanotubes used for the assembling of the sensor units where both SWCNT and MWCNT produced by the project partner SINEUROP, using arc-based methods and laser ablation for their synthesis. In the frame of the project, it has been further developed the so-called "Arc-Jet Method", where the carbon electrodes are not burned, as in conventional carbon arc synthetic route, but where a carbon feedstock gas is blown into the carbon arc. This method also allows for continuous oxidative purification of the nanotubes by blowing oxygen into the nanotube aerosol before collecting the tubes. Moreover, a special helical cold finger for collecting nanotubes by the laser ablation method has been designed and realized (Fig. 2). 


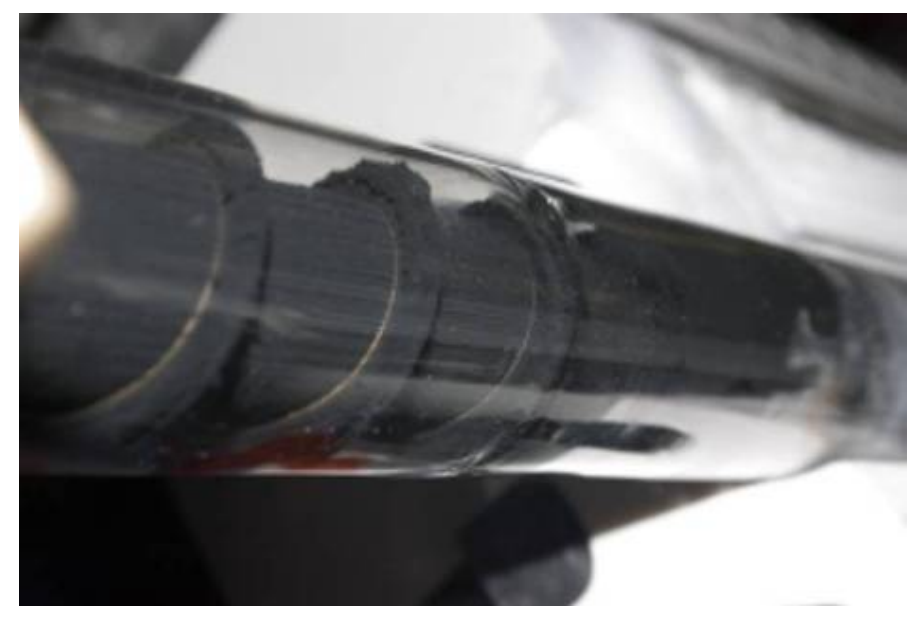

FIGURE 2. Cooled Copper Helix to Collect Nanotubes in Laser Ablation Generator.

The as-received nanotube samples were purified using $2 \mathrm{M} \mathrm{HNO}_{3}$ solutions, following procedures already settled $[3,5]$. The purified fractions were routinely analyzed by Raman micro-spectrometry and RHEED [6] in order to check phase purity and structural integrity of the material. To provide the best chances for detection of $\mathrm{NO}_{\mathrm{x}}, \mathrm{NH}_{3}$, $\mathrm{CO}$ and $\mathrm{H}_{2} \mathrm{~S}$, the most harmful gases for workers employed in constrained spaces, the as-received CNT samples were submitted to further treatments. Functionalization with organic groups, decoration with metal clusters or immersion of CNTs into conductive polymeric matrices have been studied and tested as possible solutions to improve the sensor's selectivity. All the structural, morphological and functional characterizations have been carried on the basis of protocols properly settled for the SENSATIONAL project.

The SEM images reported in figure 3 show some typical morphologies for the various CNT-based samples tested during the project.
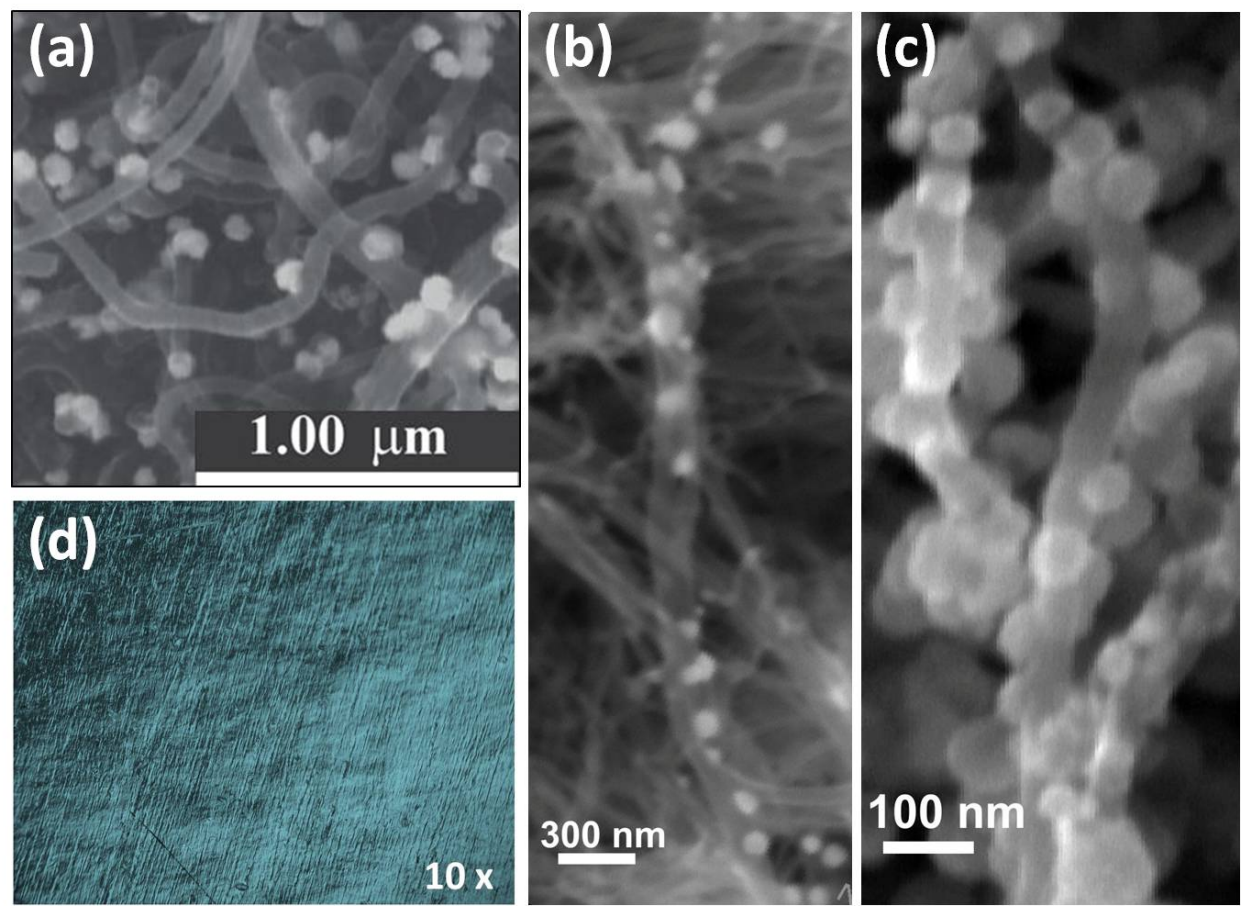

FIGURE 3. CNT-based nanomaterials with sensing capability: (a) CNT $+\mathrm{Au}$ for $\mathrm{H}_{2} \mathrm{~S}$ sensor; (b) $\mathrm{CNT}+\mathrm{Pt}$ for $\mathrm{CO}$ sensor; (c) $\mathrm{CNT}+\mathrm{Ni}$ for $\mathrm{NH}_{3}$ sensor;(d) CNT embedded in conductive polymers. 
A first sensor prototype (figure 4) has been fabricated using specifically prepared CNT dispersions onto interdigitated electrode platforms (gold electrodes with $30 \mu \mathrm{m}$ spacing, evaporated on $\mathrm{SiO}_{2}$ layer grown on $\mathrm{Si}$ substrate). A controlled volume of each dispersion was deposited between the conductive gold stripes.

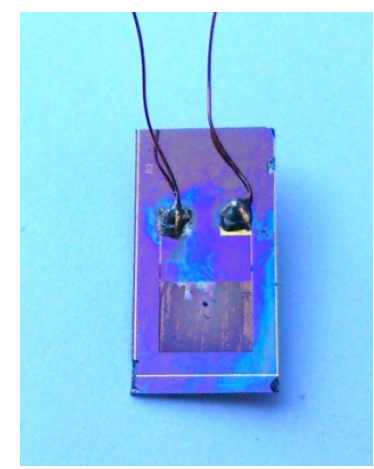

FIGURE 4. A multifinger CNT - based sensing device.

This sensor was a 3-pin device, in which the resistance is measured between drain-source pins $\left(\mathrm{R}_{\mathrm{DS}}\right)$, while the third is a gate control.

However, silicon based substrates are only partially adequate to the scope of the present project, due to the costs associated to the lithographic process for multifinger preparation, and also to the rigidity and brittleness of the resulting device. The pushing of this kind of sensors well beyond the current limits will be mainly related to the integration of the sensing elements in a textile support. Thanks to the broad experience of the project partner KLOPMAN INTERNATIONAL S.R.L. in technical textiles, we have used as support polyester/cotton mix fibers or pure multifilament polyester fibers.

The integration of the sensor with the textile has been accomplished using various existing processes, such as impregnation, rotary printing and ink printing or coating (Figure 5).
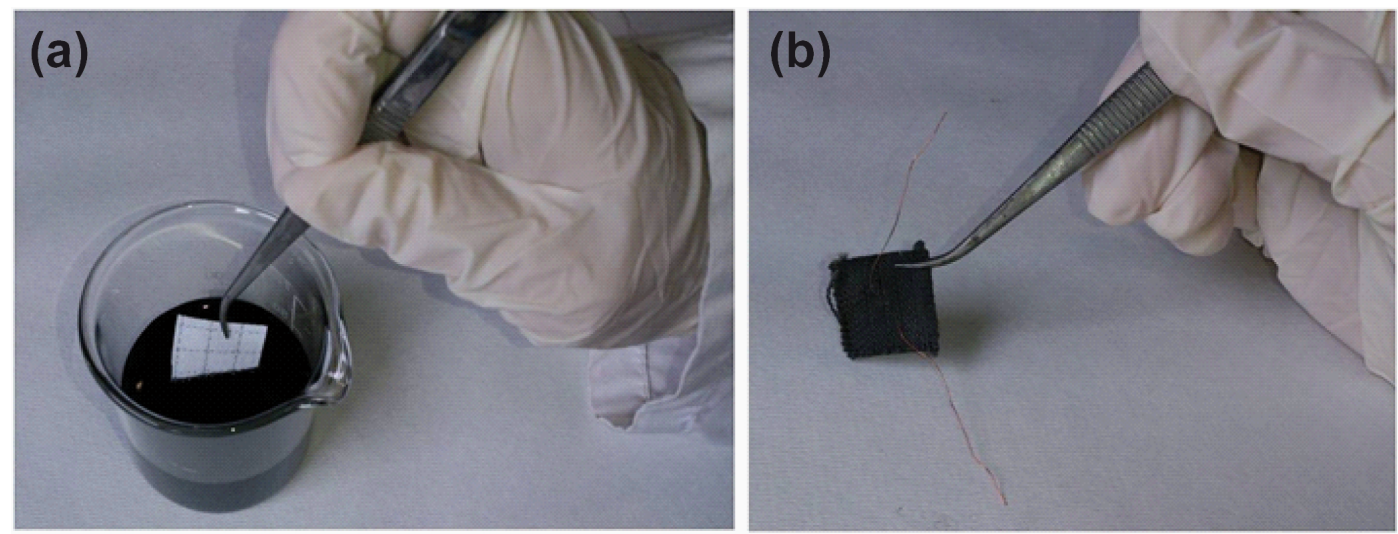

FIGURE 5. (a) Conductive textiles fabricated by dipping textile into an aqueous CNT ink followed by drying in oven at $120^{\circ} \mathrm{C}$ for $10 \mathrm{~min}$. (b) A thin, $2 \times 2 \mathrm{~cm}$ textile conductor based on a fabric sheet ( $100 \%$ cotton).

The optimization of the printing process, accomplished by adjusting the density of the material to be embedded in the tissue, represented a specific research task. The electrical circuits were made using metal wires contacting the CNT deposits by an Ag containing paint. Following the above reported fabrication scheme, a series of small (less than a squared $2 \times 2 \mathrm{~cm}$ each one) sensors supported on technical textiles have been obtained.

Typical responses of such sensor upon exposures to the various harmful gases are reported in figure 6. 

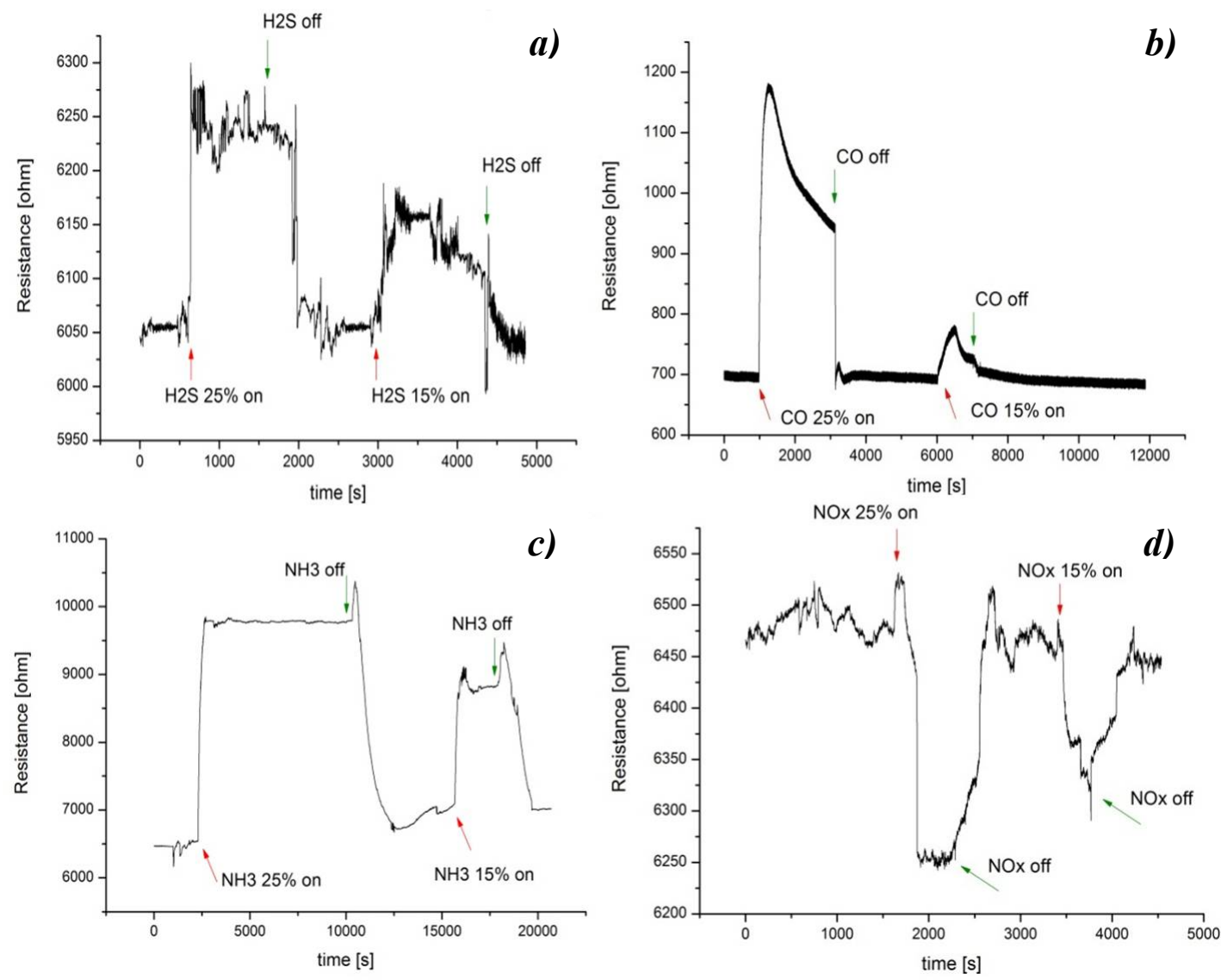

FIGURE 6. Typical response of the CNT-based textile sensors to (a) $\mathrm{H}_{2} \mathrm{~S}$, (b) $\mathrm{CO}$, (c) $\mathrm{NH}_{3}$ and (d) $\mathrm{NO}_{\mathrm{x}}$.

\section{DATA COLLECTION AND TRANSMISSION}

The use of carbon nanotubes as active material for gas detection enables to minimize size/weight and to reduce power consumption of the electronic units coupled to the sensing unit.

In our configuration the realized integrated sensor is contacted with a wearable, highly miniaturized electronic unit (less than $3 \times 2 \times 1 \mathrm{~cm}$ ). Here, the signals produced by the gas $/ C N T$ interactions are converted in numeric values and sent to the server by an ARM ${ }^{\circledR}$ Cortex ${ }^{\mathrm{TM}}-\mathrm{M} 4$ based microcontroller. The communication with server is based on wireless sensor networks using a ZigBee/IEEE RF module connective [7-9]. The whole electronic equipment is environmentally sealed, resistant to chemicals and electrically shielded. In case of any anomaly, the electronic unit is able to generate a visual and acoustic alarm. The telecommunication infrastructure was designed in order to have an appropriate communication between workers inside a confined space and the central monitoring station outside. The system, suitable for use in confined spaces, enables inter-crew communications and remote data transfer from wearable electronic units to the central monitoring station by means of the ZigBee wireless technology. A further algorithm was introduced for evaluation of the cumulative gas concentration effect measured during a mission. The algorithm is based on Bayesian Network graphical probabilistic models and it is able to understand the best action in case of an alarm event and support the decision process of the operator. Bayesian framework offers several advantages. It is simple to use, not conditioned by a long train time, efficient and flexible, and in order to be easy to extends in case of introduction of new variables it offers a compact representation of joint probability distributions and is capable of practical representation of large models, consisting of several variables [10-11]. This technology offers great flexibility in network management and uses very small amounts of power. Multi-hop routing between crew members and "range expanders" is moreover used to deliver messages to the base station outside the confined space. The use of an electro-conductive yarn connecting the detector to an external operator is proposed considering 
the possibility that radio frequency/wireless devices could not work effectively in some confined spaces such as tanks or sewers shielded by metal or concrete walls.

\section{THE PROTECTIVE CLOTH}

Figure 7 shows a put-on protective cloth with the multi-gas sensor applied near to the shoulder of the worker. The electronic unit can be suspended to the belt or housed in the pocket of the workwear. The apparel with the flexible sensing platform coupled with the light, highly miniaturized electronic unit, offers as a whole an unparalleled comfort for the operator, who will not wear any additional instrumentation and could have access to a set of functionalities that will further increase his/her safety.

The fabrication of the gas sensor can be accomplished by deploying 4 arrays of properly treated CNT directly onto selected areas of the fabric and including an electrical interface, but also using a different configuration. The full-featured, operator-friendly industrial safety detection and monitoring system can be indeed obtained also by a physical integration of apart prepared microelectronics within the workwear. In this last case, the multi-gas sensing unit, a lay-out of 4 interconnected $2 \times 2 \mathrm{~cm}$ squared textiles previously coated by the CNT-based nanomaterials, is attached by hook-and-loop fastener to the worker's apparel. As in the case of the sensor fabricated by embedding the active materials onto the fibers of the apparel, a wire makes the contact with the electronic unit. The last configuration allows for numerous washing of the protective cloth and is characterized by an easy maintenance over long time.

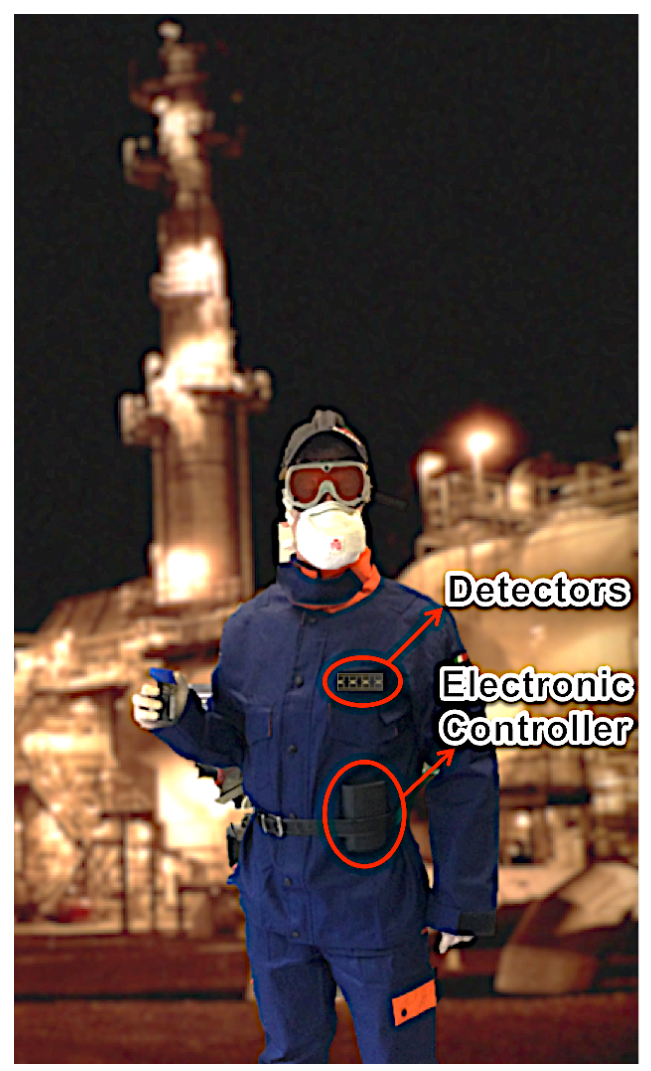

FIGURE 7. Workwear equipped with gas sensors embedded in the textile and with a wearable electronic unit. Fabrics supplied from Klopman Int: $75 \%$ cotton, $24 \%$ polyester, $1 \%$ antistatic flame retardant finished (MEGATEC 250N). 
In any case, the finishing technologies employed for the realization of the sensor ensure high flexibility and facility any contingent modification to the system's layout.

\section{MAIN OUTPUTS AND PERSPECTIVES}

The SENSATIONAL project has been brought about by the strong synergistic approach of nanotechnology and textile engineering. Nanotechnologies have been applied to give textiles the capacity to perceive chemicals present in the environment, thus realizing a new generation of reactive fibers that confer sensorial properties to the apparel used. The main technical goals reached are the manufacturing of a multi-gas sensing platform and the development of new industrial processes for integration of such a device in a protective cloth, resulting in an innovative lab-on-achip application.

The outputs of this project overcome several drawbacks of state of the art solid state gas sensors, ensuring reduction of size/weight of the device, with reduction of power consumption and of manufacturing costs.

The research areas of smart textiles and of portable gas sensors are the main fields that can take advantage of an effective production chain and of a strong industrialization plan based on the final results and on the foreseen progresses.

\section{ACKNOWLEDGMENTS}

The SENSATIONAL project was funded by the Minister for Economic Development (Italy) under the call "INDUSTRIA 2015 - Made in Italy" (contract n 00015MI01).

\section{REFERENCES}

1. F. Toschi, V. Guglielmotti, S. Orlanducci, E. Tamburri, I. Cianchetta, D. Sordi, M. L. Terranova, M. Lucci, M. Rossi, A. Andretta, P. De Stefanis, Nanotec IT Newsletter 12, 26-28 (2011).

2. M. Lucci, P. Regoliosi, A. Reale, A. Di Carlo, S. Orlanducci, E. Tamburri, M. L. Terranova, C. Di Natale, A. D’Amico, R. Paolesse, Sensor. Actuat. B-Chem. 111-112, 181-186 (2005).

3. M. L. Terranova, M. Lucci, S. Orlanducci, E. Tamburri, V. Sessa, A. Reale, A. Di Carlo, J. Phys.-Condens Mat. 19, 225004 (2007).

4. M. Lucci, F. Toschi, V. Guglielmotti, S. Orlanducci, M. L. Terranova, Journal of Sensors 2012, 359271 (2012).

5. D. Passeri, M. Rossi, A. Alippi, A. Bettucci, M. L.Terranova, E. Tamburri, Physica E 40, 2419-2424 (2008).

6. M. Rossi, D. Manno, S. Orlanducci, A. Serra, M. L. Terranova, "Electron diffraction analysis of carbon-based nanomaterials" in Encyclopedia on Nanoscience and Nanotechnology 13, edited by H. S. Nalwa, 2011, pp. 375-425.

7. E. Castillo, Neural Process. Lett. 7, 151-159 (1998).

8. V. K. David and S. Rajasekaran, "Pattern Recognition Using Neural and Functional Networks" in Studies in Computational Intelligence 160, Berlin Heidelberg: Springer-Verlag, 2009.

9. E. A. El-Sebakhy, A. S. Hadi, K. A. Faisal, IEEE T. Neural Networ. 18, 843-850 (2007).

10. N. Friedman, D. Geiger, M. Goldszmidt, Mach. Learn. 29, 131-163 (1997).

11. F.V. Jensen, Bayesian Networks and Decision Graphs, Springer-Verlag, 2007. 